\title{
Digital publishing in the faculties of arts and humanities: prospects, chances, and risks
}

\author{
Georg Kamp
}

Published online: 6 February 2008

(C) Springer-Verlag 2008

As access to the internet has become absolutely common and data throughput has increased enormously by new transmission techniques, online accessible texts have more and more become an alternative to scientific publishing in books, anthologies, and journals. Texts that are openly accessible in online journals, forums for discussions, on university servers or on the personal homepage of a scientist are immediately available to everybody. Publishers and libraries that traditionally have played the central part in organizing the communication of the scientific community are principally open to this change-however, to a very differing extent; they supply their specific competences to related processes, whereas their influence on availability and usage of scientific texts decreases.

However, it is uncertain in how far the community should leave the progress of change to the decentralized and rather uncoordinated developments of the internet and the initiative of a few "avant-gardists": in order to guarantee a successful scientific communication, the texts do not only have to be made accessible, they also have to be retrieved systematically - and for as long a time as possible. In view of the accumulating masses of data and information, a reliable, non-selective and transparent supply and storage of data is necessary. Moreover, free availability of neutral-especially not ideologically biased-research instruments are to be guaranteed.

At the same time the desideratum for efficient procedures to assess-with respect to pure disciplinary measures - the relevance and validity of online accessible texts increases with their quantity. In the tradition of scientific publishing, a highly complex system of reputation ascription (e.g. peer reviewing) and reputation documentation (e.g. place of publication) has been developed. Nowadays not only is the text-based research of the academic recipients widely dependent on these means,

G. Kamp $(\bowtie)$

Europäische Akademie Bad Neuenahr-Ahrweiler GmbH,

Bad Neuenahr-Ahrweiler, Germany

e-mail: georg.kamp@ea-aw.de 
but also the reputation of the authors in the community, with corresponding consequences for recruiting and staffing procedures. Such a system is hardly developed yet for digital publishing, and it is at least questionable whether it can be reproduced under the different conditions of digital communication.

But it is also uncertain whether it should be reproduced in the same way. Changed publishing conditions in digital media offered some new options that hitherto have not been available by publications in books and journals: digital storage and hyperlinks enable to amend contents and to bring them into new contexts. This does not only support a faster and more systematic supply of information, it also allows for subsequent commenting on already published texts at the same place. For example, the comment stating that the repetition of a certain experiment has not led to the same results can be immediately "added" to the article that describes it for the first time. And the comment that a certain interpretation of a classical citation holds plausible only by disregarding its immediate context can be attached directly to its proposition. In traditional publishing organs, commentaries would have been mentioned in some later edition of the same or another journal, only perceptible by chance or for those who invest a lot of time in systematic scans of their discipline's periodicals.

In the meantime, various validation methods adequate to the digital medium became established along the anonymous communication requirements of the internet (see for example the method of evaluated short reviews on the pages of online booksellers). Varieties of these methods are already accepted and used for scientific communication in different ways.

Consequently, a change in the style of publication can also be observed: the texts often become shorter and of a more temporary kind and-compared to the articles submitted to printed media-understand themselves as contributions to ongoing discussions, which will promote, but not decide the scientific debate. Therefore, according to observers, research activities within the scientific community as a whole are increasingly determined by the character of a (disciplinary as well as trans-disciplinary) "team work" among the scientists of globally scattered institutes. So, the academic and scientific communication in the internet brings about new and promising possibilities, but it generates new desiderata regarding cognitive authorship, copyright, and reputation, among others. ${ }^{1}$

Furthermore, a change in reception strategies may be expected: on the one hand the opportunity of full-text search in books and journals, which is presently extended, e.g. by "Google Book Search" and "Google Scholar" as well as by the databases supplied by libraries, offers a more systematic access to scientific publications-as has already been mentioned before. On the other hand-in particular under the present coexistence of printed and online publications-there is a distortion to be seen: according to a study initiated by the Deutsche Forschungsgemeinschaft, online accessible media are not as acceptable by the experts as the printed ones, but at the same time they are to be found in the totally quoted literature to an extraordinarily high extent. ${ }^{2}$ From this observation it is to be assumed that the amounts of quotation

\footnotetext{
${ }^{1}$ Giles J (2006) The trouble with replication. Nature 442:344-347.

2 Deutsche Forschungsgemeinschaft: Publikationsstrategien im Wandel? Ergebnisse einer Umfrage zum Publikations- und Rezeptionsverhalten unter besonderer Berücksichtigung von Open Access. Bonn 2005.
} 
have to be seen rather in proportion to the efforts of finding and consulting the texts than in proportion to their quality.

Up to now, especially in the faculties of arts and humanities, there is only a small recognition of the changing cultures of publishing and reception. The form of publication mostly associated with these disciplines-the elaborated monograph, traditionally published as a comprehensive article or book-is very far away from those organizational principles of digitalized documentation that offer added value. Hence, it is no surprise that the members of these disciplines are "conservative" with respect to their devotedness to printed media. Nevertheless, especially texts of this kind might profit from receptions supported by digital devices, either by offering the possibility for fast finding of certain topics or definitions by simple fulltext search (which may supplement or substitute any indices), or by enabling more complex analyses by means of appropriate software tools.

Meanwhile, the development of manner and standards of digital publishing is promoted far more actively by natural scientists, according to the quite different requirements for communication in their disciplines. With regard to the unquestionable positive results in these areas, scientific administrators and funding agencies support this progress for a number of reasons: from a certain "democratisation effect" of research results being obstacle-free accessible for everyone to expected cost reduction potentials of digitalised scientific communication. But only if the changes in the communication infrastructure in arts and humanities meet the specific requirements of the scholars and if the adverse effects of this transition can be avoided, a benefit from the new techniques could be expected.

In order to contribute to the debate on an active shaping of the future communication infrastructure of these disciplines, the Europäische Akademie Bad Neuenahr-Ahrweiler GmbH held a symposium in spring 2007 on this topic. The following section will give a survey of some central points of the given papers and the discussion.

Wulf D. von Lucius, an economist and German publisher (Lucius\&Lucius Verlagsgesellschaft $\mathrm{mbH}$ ), focuses directly on the requirements of a functioning scientific communication system, in how far the established publishing houses can contribute to it and which (monetary and other) risks are connected with the alternatives. He specifically compares the pros and cons of the discussed strategies for digitalisation and argues for the so-called "green road" where the management of digital publishing remains in the hand of the publishers.

The "Forum Qualitative Sozialforschung/Forum: Qualitative Social Research" (FQS) is an example for an operative open access journal, which is managed by scientists themselves. How it was established, how it works, and which efforts and chances are connected with this option is shown by the relevant paper of Katja Mruck and Günter Mey.

The technical conditions of functioning and functional usage of digitalised texts in the humanities are subject of the contribution of Wilhelm Ott (Professor emeritus at the Eberhard-Karls-University of Tübingen). If publishing should reach its public over long time periods, the problem of long-term storage of digital data in platformand media-independent formats turns out to be crucial. The problem is not yet solved in a sufficient manner. How a promising strategy would look like, is the main 
topic of the first part of his paper. Furthermore, if digitally published texts should not only be an equivalent alternative to printed ones but also useful resources for special investigation features of digital ("data-processing") devices, they have to be formatted and arranged in an adequate manner. In the second part of his paper, the author demonstrates how this could look like generally, and how it looks like in case of the "TUebingen System of Text Processing programs" (TUSTEP).

Texts are the "communication-tools" of the humanities, and at the same time their primary object of interest. From this perspective, Stefan Gradmann and Jan Christoph Meister (both from the University of Hamburg) take a closer look on the change from "analogue" to "digital representation mode". With respect to C.P. Snows' hypothesis on "The Two Cultures", they expect a triple paradigm shift going along with the "digital turn" in the humanities and the social sciences, that can, under certain preconditions, bring about an additional specific cognitive value of the systematic usage of digital texts. 